\title{
Effectiveness of Pranayama (Yogic Breathing Exercise) on Management of Somatosensory Tinnitus: A Single Case Study
}

\author{
${\text { Badariya } \mathbf{M}^{1} \text {, Madhavan Balakrishnan }}^{2}$, Sithara Shanavas ${ }^{3}$ \\ 1,2Associate Professor, Department of ASLP, AWH Special College, Kallai, Calicut, Kerala, India. \\ ${ }^{3}$ II MASLP Student, Department of ASLP, AWH Special College, Kallai, Calicut, Kerala, India.
}

Corresponding Author: Badariya M

\begin{abstract}
Somatic or somatosensory tinnitus is a subtype of subjective tinnitus, in which tinnitus perception is caused by an alteration in somatosensory afference from the cervical spine or temporo-mandibular area which changes patient's tinnitus perception in terms of loudness, pitch and localization. A 30year-old female reported with the complaint of tinnitus, vertigo and reduced hearing sensitivity in the left ear for 7 months. Enduring low frequency annoying tinnitus flare ups with vertigo, vomiting and speech understanding difficulty every 48-72hours. Successive audiological investigations and THI score revealed mild sensorineural hearing loss in the left ear with grade 5 catastrophic handicap. Tinnitus retraining Therapy (TRT) along with pranayama (yogic exercise) was used as a treatment protocol. Nadi Shodhana (Alternate-Nostril Breath- one of the most efficient strategies for calming nerves and settling mind fluctuations) was practised twice daily and during the treatment sessions. Effectiveness of TRT combined with Yoga on a patient with severe tinnitus \& accompanying issues was investigated. This treatment strategy proved to be highly effective in lowering tinnitus severity and reducing the accompanying issues with long term effectiveness. As a result, present study enlightens the possible success rate of this treatment protocol for somatosensory tinnitus and its impact on tinnitus recurrence.
\end{abstract}

Key Words: Somatosensory tinnitus, TMJ, Pranayama, TRT, Catastrophic handicap.

\section{INTRODUCTION}

Somatic or somatosensory tinnitus (ST) is a subtype of subjective tinnitus, ${ }^{[1]}$ in which tinnitus perception is caused by an alteration in somatosensory afference from the cervical spine or temporomandibular area, causes or changes a patient's tinnitus perception. [2] This means that the psychoacoustic attributes of tinnitus (loudness, pitch and localization) might be changed immediately (though only temporarily) by different stimuli, such as forceful muscle contractions of head, neck and limbs [3-7]; eye movements in the horizontal or vertical axis, ${ }^{[8,9]}$ pressure on myofascial trigger points ${ }^{[10,11]}$; as well as orofacial movement. ${ }^{[12]}$ This modulation is very prevalent for $65-80 \%$ of patients with tinnitus. ${ }^{[2,13]}$ Individuals with ST frequently report sleep disorders, concentration and attention problems, and symptoms of anxiety and/or depression. Due to a lack of unambiguous clinical tests, many authors and clinicians use their own criteria for diagnosing ST. This resulted in large differences in prevalence of ST, which vary from $16 \%$ to $83 \%$ in different studies ${ }^{[2,14]}$ and limits the comparison of clinical trials on ST treatment. ${ }^{[11,15]}$ the presence of symmetric pure tone thresholds in both ears (normal or abnormal) and unilateral or asymmetric tinnitus should direct the patient 
Badariya M, Balakrishnan M, Shanavas S. Effectiveness of pranayama (yogic breathing exercise) on management of somatosensory tinnitus: a single case study.

towards a more detailed evaluation of somatosensory tinnitus. A set of Diagnostic criteria proposed by Sanchez and Rocha (2011) helps recognize patients with ST in clinical practice. Several studies have found connections between the somatosensory system of the cervical or temporomandibular area and the cochlear nuclei (CN), offering a physiological explanation for ST. ${ }^{[10-17]}$

Lack of unique treatment being effective for $100 \%$ of patients, various treatment approaches of tinnitus the have been modified to include patients with somatosensory tinnitus. These guidelines recommend the combined use of a specific protocol and choosing adequate therapeutic options that will target auditory pathways and musculoskeletal disorders may increase the possibility of satisfactory results. [14] The more recent modes of treatment focus on application of yoga and meditation in management of tinnitus along with regular otological and audiological treatment options. ${ }^{[19]}$ Yoga is commonly known for its relaxation effect, there are various styles of yoga [20] however, Pranayama in particular will be explored, Pranayama is a Sanskrit word which can be broken down into two parts: "Prana (means vital force) and Yama (means control)". There are different styles of pranayama, which vary "based on the breathing cycles, tidal volume and other factors like the use of mouth, nostrils, constriction of laryngeal muscles, and position of the glottis". By controlling one's breathing, one can improve their cardiovascular health, respiratory function, and can facilitate in decreasing stress. Continued use of this practice can reduce the autonomic system's sympathetic response, leading to increased relaxation and decreased stress levels. ${ }^{[19]}$ The present case highlights effectiveness of pranayama combined with audiological management of somatosensory tinnitus caused due to TMJ problem which is a consequence of dental surgery. This will primarily demonstrate how sound therapy and pranayama can aid in the alleviation of unpleasant tinnitus and its consequences.

\section{CASE REPORT History}

A 30-year-old female referred by an ENT surgeon reported to the department of ASLP with the complaint of tinnitus, vertigo and reduced hearing sensitivity in the left ear for 7 months. The client was interviewed for detailed case history and revealed that the enduring tinnitus progresses often with vertigo, vomiting and speech understanding difficulty. Enduring low frequency annoying tinnitus flare ups with alternating pitch, vertigo, vomiting, severe headache, heaviness of head and speech understanding difficulty every 48-72hours.The client underwent a dental surgery 1 year ago and had been on medicine for a few months. Onset of tinnitus and associated complaints were apparent followed by the dental surgery, at this point of time client was referred to an ENT. Client was also experiencing sleep disturbances (fragmented sleep) and intolerance to loud noises such as cooker whistle, heavy traffic, and machinery noises. This resulted in mental and emotional anguish, which interrupted her personal and social well-being and caused her to avoid social gatherings and public locations. Client was on vertigo medication (tab Vertin) and was referred to the neurology and audiology department by an ENT.MRI SCAN recommended by Neurologist which discarded the existence of lesions as per patient's complaints.

\section{Audiological Assessment}

The client underwent routine audiological assessments, including pure tone audiometry, speech audiometry, and immittance evaluations. Audiometric test was performed utilizing an MAICO MA 42 clinical audiometer and supra-aural headphones. The results showed that the left ear had a mild degree of hearing loss (30 $\mathrm{dB})$ and the right ear had normal hearing sensitivity (11.25 dB), with $100 \%$ speech identification scores and >100dBHL 
Badariya M, Balakrishnan M, Shanavas S. Effectiveness of pranayama (yogic breathing exercise) on management of somatosensory tinnitus: a single case study.

uncomfortable loudness levels in both ears [FIGURE1]. A GSI Tympstar pro instrument was used to assess immittance, and the results revealed "A" type tympanogram for both ears, with ipsilateral and contralateral reflexes. As a result, a provisional diagnosis of mild sensorineural hearing loss in the left ear and normal hearing sensitivity in the right ear was made.

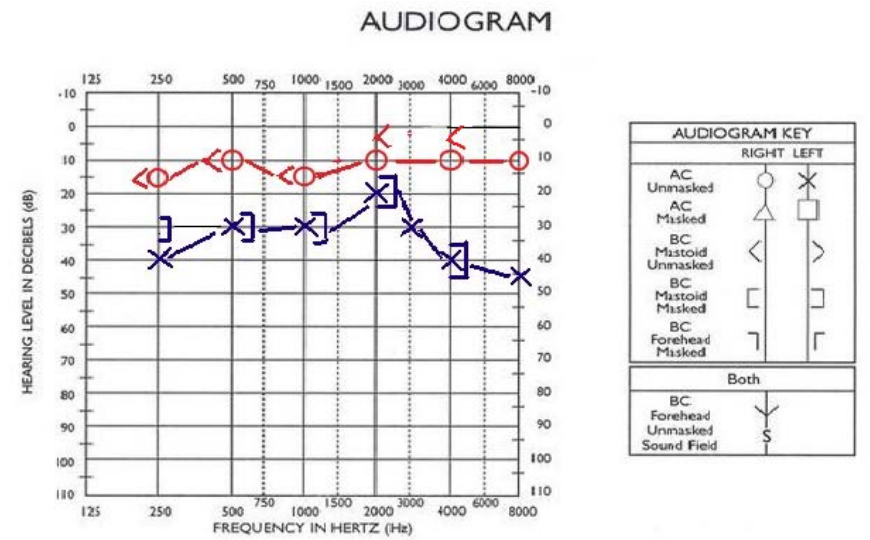

Figure 1: Audiogram of client during the initial evaluation.

Further, MAICO ERO-SCAN was used to measure bilateral DPOAEs to check outer hair cell function; the absence of OAEs in the left ear indicated OHC dysfunction. Short Increment Sensitivity Index (SISI), Tone Decay Tests (TDT), Supra Threshold Adaptation Tests (STAT), and Reflex Decay Tests were among the special tests used to rule out the presence of cochlear and retro cochlear involvement (RDT). None of the tests have turned up significant that could have contributed to the patient's diagnosis.

After normal audiological assessments, the client was given the Tinnitus Handicap Inventory, and she received 82 points, placing her in the grade five group, which was interpreted as a catastrophic handicap. Tinnitus was assessed using MAICO MA 42 clinical audiometer with circumaural headphones. The pitch of the tinnitus was matched with a Narrow band noise (NBN) of $6 \mathrm{KHz}$ centre frequency, which was used as a reference for the loudness match, using two alternative forced choice pitch matching procedures. $6 \mathrm{KHz} \mathrm{NBN}$ was presented at a level just below the threshold (20dBHL) for loudness matching, and intensity was increased until the subject signalled a match, which was around 30-35dBHL.
Residual inhibition was carried out for BBN at $45 \mathrm{dBHL}$ and client reported reduction in tinnitus loudness for a brief period of time. This finding was interpreted as client could benefit from tinnitus retraining therapy (TRT).

\section{Audiological Management}

Breaking the conventional treatment scenarios decision was made upon focusing on the associated problems which was the major concern. Decision was made upon the implementation of pranayama with specific treatment protocol such as Tinnitus retraining Therapy (TRT). During the initial phase of treatment, patient was introduced to TRT, because the client was not able to concentrate on her breathing which in turn relaxes and decreases stress levels due to the presence of annoying tinnitus. TRT sessions were scheduled for $40 \mathrm{~min}$, 5days/week were utilized for counseling followed by sound therapy. Each treatment session was planned in such a way that, 1 min BBN exposure at 45-60 dBHL followed by habituation and counseling for a total of 3 sets/session. This was done for two weeks to relax the patient and urge her to participate actively in the therapy sessions. Frequency of tinnitus flare ups every 48 hours lasted for 15-20 minutes during this period. Tinnitus loudness and 
Badariya M, Balakrishnan M, Shanavas S. Effectiveness of pranayama (yogic breathing exercise) on management of somatosensory tinnitus: a single case study.

bouts of tinnitus flare-ups (twice/week) were reduced after two weeks of TRT. The same treatment protocol was followed for another week which resulted nonappearance of tinnitus for 2-3 hours. The client's drive and confidence were boosted as a result of this phenomenon.

Client was introduced to Nadi Shodhana during the third week of TRT by making her sit on a chair with spine erect posture and was taught to take the air through the right nostril and release it through the left nostril after closing the right nostril with the thumb which completes one single round. Initially client was taught the above without the retention of breath (kumbhaka). When the client was comfortable with this practice, kumbhaka was started by retaining the breath for 5 secs after every inhalation and gradually the duration was increased up to 30 secs. During this time speed of inhalation and exhalation was very gentle. Nadi Shodhana was practised twice daily and she was advised to perform the same whenever needed (during/before tinnitus flare-ups). Each Nadi Shodhana session last 5-10 minutes or until the client attained complete relaxation. Single treatment session consisted of Nadi Shodhana, TRT followed by counselling. At the same time, the patient was taught several coping mechanisms and tactics that might be used during tinnitus flare-ups, such as listening to soothing music, birds singing, leaves rustling, and so on. Additionally, the client was recommended to continue the use of ear plugs while travelling or exposed to loud noises.

The THI was re-administered after 34 sessions of TRT and 5 weeks of pranayama, and the results showed that the severity of tinnitus had decreased, with scores falling into the grade 2 category, indicating a mild impairment. Audiological investigations were repeated and the audiometric thresholds remained the same for both ears. Low-frequency continuous tinnitus became soft intermittent tinnitus (appearing two or three times a week and lasting only for a few hours). Vertigo and vomiting episodes subsided, and she was able to resume a normal sleep cycle. In order to avoid a sudden tinnitus flare-up, the use of ear plugs was continued in unfavourable situations. Of the below figures, Figure 2 represents TRT scores across the treatment period and Figure 3 depicts the reduction in tinnitusrelated/associated difficulties as a result of treatment.

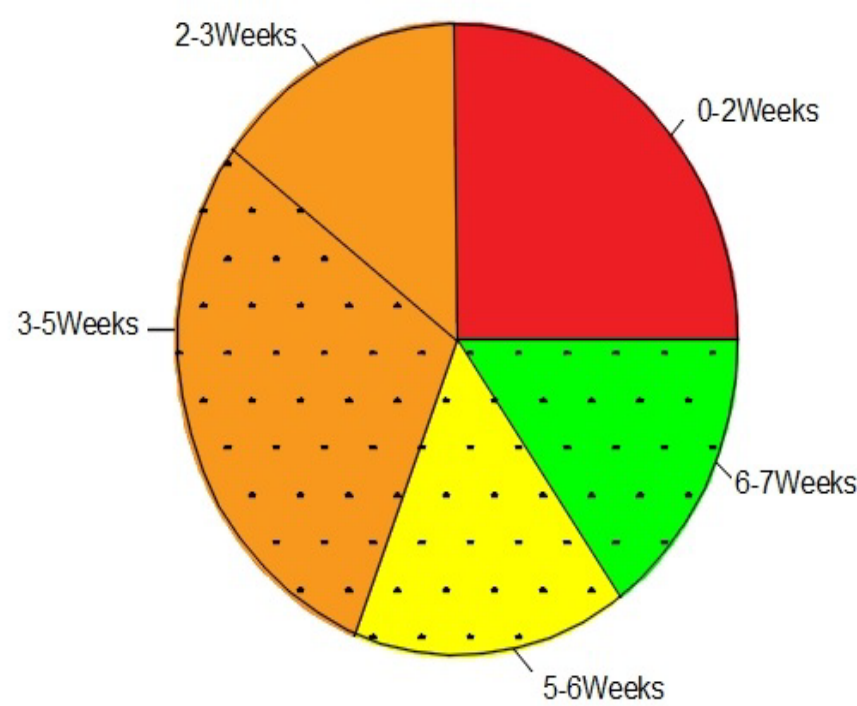

Mild handicap(Grade2)

Moderate handicap(Grade3)

Severe handicap(Grade4)

Catastrophic handicap(Grade5)

$\boxplus$ TRT incorporated with breathing exercises

Figure2: THI scores across the periods of treatment. 


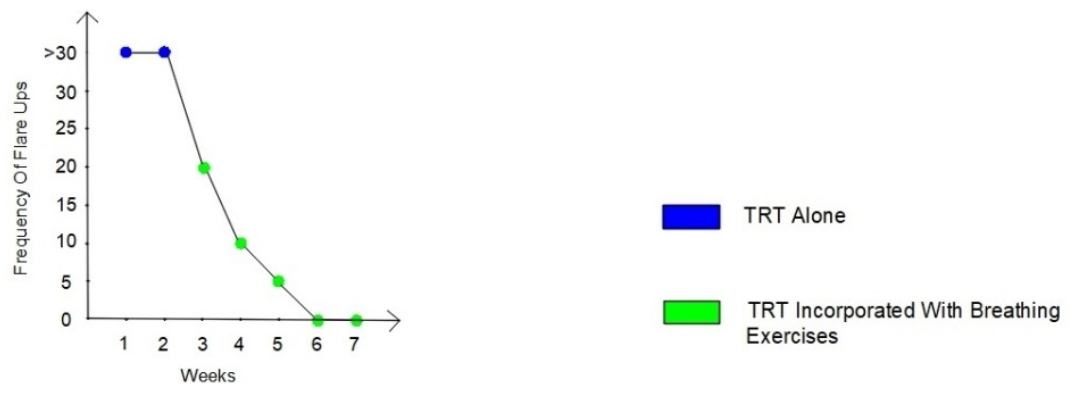

Figure 3: Effect of treatment on recurrence of associated problems.

\section{DISCUSSION}

Studies have been pointing towards a high prevalence of somatosensory tinnitus among tinnitus patients, it is found that TMJ is the area most often responsible for fluctuations in tinnitus loudness followed by the head and neck area. ${ }^{[21-23]}$ As a proxy for stereotyping traditional treatment strategies, we combined conventional sound therapy, counseling and pranayama. As reported by literature that both existing and novel tinnitus treatments, no one treatment is currently available that is effective for all tinnitus sufferers. ${ }^{[24,25]}$ This method not only abridged the symptoms but also the chances of recurrence also minimized as pranayama exercise became a part of her new lifestyle.

During the first phase of treatment, TRT was effective in reducing the loudness and duration of tinnitus. This was assessed based on the percentage of time that participants were aware of their tinnitus, were annoyed by their tinnitus, and on an analysis of scores from the THI. [24, 25] During this phase, awareness was present most of the time, annoyance was limited to quieter environments and TRT scores are represented in figure 2.

Even though reduction in loudness of tinnitus was achieved via TRT, limited benefit was obtained on reducing associated problems. While TRT has been proven beneficial for many tinnitus sufferers, it cannot be definitively said that it trumps other forms of tinnitus treatments in all cases. ${ }^{[26]}$ Tinnitus reactions and associated problems are known to be linked to stress and anxiety, [27] hence we aimed to investigate the effectiveness of pranayama in this particular client in subsiding the associated problems. However, it is not possible to conclude that the success of treatment ${ }^{[24-26]}$ will be similar across large population because lack of significance due to the single case study. As a result, future research can examine the likely success rate of this therapy strategy. It's critical to keep researching on this subject to see how effective sound therapy paired with pranayama can be. The impact of this treatment paradigm on tinnitus recurrence should also be looked into further.

\section{CONCLUSION}

Nadi shodhana pranayama create a deep sense of well-being and harmony on the physical, mental, and emotional levels and help with headaches, migraines, and other stress-related symptoms. ${ }^{[26]}$ It was noted that there are very few scientific articles discussing the physiological benefit of pranayama on patient with tinnitus. Hence, in this case study effectiveness of pranayama combined with TRT was investigated on a patient with severe tinnitus and accompanying issues. This treatment strategy proved to be highly effective in 
Badariya M, Balakrishnan M, Shanavas S. Effectiveness of pranayama (yogic breathing exercise) on management of somatosensory tinnitus: a single case study.

lowering our client's tinnitus severity and reducing the accompanying issues with long term effectiveness.

\section{Acknowledgement: None}

\section{Conflict of Interest: None}

\section{Source of Funding: None}

\section{REFERENCES}

1. Levine RA. Somatic (craniocervical) tinnitus and the dorsal cochlear nucleus hypothesis. Am J Otolaryngol. 1999 NovDec;20(6):351-62

2. Michiels S, Ganz Sanchez T, Oron Y, Gilles A, Haider HF, Erlandsson S etal., Diagnostic Criteria for Somatosensory Tinnitus: A Delphi Process and Face-toFace Meeting to Establish Consensus. Trends Hear. 2018 Jan-Dec; 22:2331216518796403.

3. Levine, R. A. (1999, September). Somatic modulation appears to be a fundamental attribute of tinnitus. In Proceedings of the Sixth International Tinnitus Seminar (pp. 193-197). London: The Tinnitus and Hyperacusis Center.

4. Sanchez TG, Guerra GCY, Lorenzi MC, Brandão AL, Bento RF. The influence of voluntary muscle contractions upon the onset and modulation of tinnitus. Audiol Neurootol. 2002;7:370

5. 10.1159/000066155.

5. Sanchez TG, da Silva Lima A, Brandão AL, Lorenzi MC, Bento RF. Somatic modulation of tinnitus: test reliability and results after repetitive muscle contraction training. Ann Otol Rhinol Laryngol. 2007 Jan;116(1):305.

6. Sanchez TG, Guerra GCY, Lorenzi MC, Branda o AL, Bento RF. The influence of voluntary muscle contractions upon the onset and modulation of tinnitus. Audiol Neurootol. 2002;7:370-5

7. Sanchez TG, Lima AS, Brandao AL, Lorenzi MC, Bento RF. Somatic modulation of tinnitus: test reliability and results after repetitive muscle contraction training. Ann Otol. 2007;116:30-5.

8. Coad, M. L., Lockwood, A., Salvi, R., \& Burkard, R., Characteristics of patients with gaze-evoked tinnitus. Otology \& Neurotology, 22(5). 2001; 650-654.
9. Baguley DM, Phillips J, Humphriss RL, Jones S, Axon PR, Moffat DA. The prevalence and onset of gaze modulation of tinnitus and increased sensitivity to noise after translabyrinthine vestibular schwannoma excision. Otol Neurotol. 2006; 27:220-4

10. Rocha CA, Sanchez TG. Myofascial trigger points: another way of modulating tinnitus. Prog Brain Res. 2007; 166:209-14.

11. Bezerra Rocha CA, Sanchez TG, Tesseroli de Siqueira JT. Myofascial trigger point: a possible way of modulating tinnitus. Audiol Neurootol. 2008;13(3):153-60.

12. Pinchoff RJ, Burkard RF, Salvi RJ, Coad ML, Lockwood AHAm J. Modulation of tinnitus by voluntary jaw movements. Otol. 1998 Nov; 19(6):785-9.

13. Shore S, Zhou J, Koehler S. Neural mechanisms underlying somatic tinnitus. Prog Brain Res. 2007;166:107-23. 10.1016/S0079-6123(07)66010-5

14. Ganz.S. T, Rocha.C.B. Diagnosis and management of somatosensory tinnitus. Clinics 66.6 2011; 1089-1094.

15. Abel M. D., Levine R. A. (2004) Muscle contractions and auditory perception in tinnitus patients and nonclinical subjects. Cranio 22(3): 181-191.

16. Levine R. A., Abel M., Cheng H. (2003) CNS somatosensory-auditory interactions elicit or modulate tinnitus. Experimental Brain Research 153(4): 643-648.

17. Michiels S., De Hertogh W., Truijen S., Van de Heyning P. (2015) Cervical spine dysfunctions in patients with chronic subjective tinnitus. Otology \& Neurotology 36(4): 741-745.

18. Michiels S., Van de Heyning P., Truijen S., Hallemans A., De Hertogh W. (2017) Prognostic indicators for decrease in tinnitus severity after cervical physical therapy in patients with cervicogenic somatic tinnitus. Musculoskeletal Science \& Practice 29: 3337.

19. Kuppusamy M, Kamaldeen D, Pitani R, Amaldas J, Shanmugam P. Effects of Bhramari Pranayama on health - A systematic review. J Tradit Complement Med. 2017 Mar 18;8(1):11-16.

20. Köksoy S, Eti CM, Karataş M, Vayisoglu Y. The Effects of Yoga in Patients Suffering from Subjective Tinnitus. Int Arch Otorhinolaryngol. 2018;22(1):9-13. doi:10.1055/s-0037-1601415 
Badariya M, Balakrishnan M, Shanavas S. Effectiveness of pranayama (yogic breathing exercise) on management of somatosensory tinnitus: a single case study.

21. Ralli, M., G. Altissimi, R. Turchetta, and G. Cian-frone. Somatic modulation of tinnitus: a review and some open questions. Otolaryngol Open J 2, no. 4 (2016): 111-114.

22. Ralli, M., Greco, A., Turchetta, R., Altissimi, G., de Vincentiis, M., Cianfrone, G. (2017) Somatosensory tinnitus: Current evidence and future perspectives. Journal of International Medical Research 45(3): 933947.

23. Mc Ferran DJ, Stockdale D, Holme R, Large CH, Baguley DM. Why Is There No Cure for Tinnitus? Front Neurosci. 2019 Aug 6; 13:802.

24. Henry JA, Schechter MA, Zaugg TL, Griest S, Jastrebof H PJ, Vernon JA, et al. Clinical trial to compare tinnitus masking and tinnitus retraining therapy. Acta OtoLaryngological 2006;126:64-9.

25. Henry JA, Schechter MA, Zaugg TL, Griest S, Jastreboff PJ, Vernon JA etal., Outcomes of clinical trial: tinnitus masking versus tinnitus retraining therapy. J Am Acad Audiol. 2006 Feb;17(2):104-32.

26. Sabeena A. Ramnanan. The Power of a Sound Mind: Exploring Meditation and Sound Therapies for Treating the Emotional Impact of Tinnitus. The Graduate Center, City University of New York 6,2021.

27. Vanneste, S., Plazier, M., der Loo, E. van, de Heyning, P. V., Congedo, M., \& De Ridder, D. (2010). The neural correlates of tinnitus-related distress. NeuroImage, 52(2), 470-480.

How to cite this article: Badariya $\mathrm{M}$, Balakrishnan M, Shanavas S. Effectiveness of pranayama (yogic breathing exercise) on management of somatosensory tinnitus: a single case study. Int J Health Sci Res. 2021; 11(12): 96-102. DOI: https://doi.org/10.52403/ijhsr. 20211215 